\title{
Interresponse time reinforcement: Transition and steady states'
}

\author{
Riehard W. Malott 2 and William W. Cumming \\ COLUMBIA UNIVERSITY
}

\begin{abstract}
Abstraet
A schedule of reinforcement was used in which reinforcement was delivered to only those responses with associated interresponse times (IRTs) falling within a prescribed temporal interval. For each of three different groups of rats, a different value was used for the lower bound of the reinforcement interval. In all cases, the width of the interval was equal to the lower bound. This schedule was put into effect following regular reinforcement of all IRTs. The probability of shortIRTs, and the mode and interquartile range of the IRT distributions were computed as a function of the number of sessions exposure to the IRT reinforcement schedule.
\end{abstract}

\section{Problem}

For a variety of reasons, much of the recent work on schedules of reinforcement has been concerned with the steady state performance of the organism when exposed to these schedules; the transitional behavior has, to some extent, been ignored. The rationale behind steady state research has been well presented by Sidman (1960). The validity of using a "stability criterion" to determine the point at which steady state has been obtained has been seriously questioned by Cumming \& Schoenfeld (1960). An alternative to the stability criterion is the extended exposure of Ss to a single value of a reinforcement schedule. Data obtained from these Ss may then be used as a baseline against which other Ss may be compared. Once Ss have been exposed well beyond the point where stability has been reached, it should be much easier to establish some conservative value beyond which stability will surely have been obtained. This value can then be used instead of the stability criterion.

\section{Method}

Six naive male albino rats were used. They were three months old at the onset of the experiment. One week before the beginning of the experiment and continuing throughout the study, the animals were maintained at $80 \%$ of their extrapolated normal weight. They were fed at the end of each daily experimental session and once each day on weekends when experimental sessions were not conducted.

A single-lever Grason-Stadler test chamber was modified by the addition of a liquid dipper. The dipper arm, normally within the cage, could be momentarily lowered into the reservoir and returned to deliver a drop of Eagle Brand sweetened milk. The programing and recording equipment was housed in an adjoining room.

Five daily sessions of $50 \mathrm{~min}$. duration were used for dipper training, and successive approximation and
Table I. Procedure.

$\begin{array}{lcccc}\text { Rats } & \begin{array}{c}\text { Lower } \\ \text { Bound } \\ \text { (sec) }\end{array} & \begin{array}{c}\text { Interval } \\ \text { Width } \\ \text { (sec) }\end{array} & \begin{array}{l}\text { Session } \\ \text { Length } \\ \text { (min) }\end{array} & \begin{array}{c}\text { IRT Class } \\ \text { Width } \\ \text { (sec) }\end{array} \\ 76,77 & 4 & 4 & 25 & 0.5 \\ 72,73 & 8 & 8 & 50 & 0.5 \\ 66,67 & 16 & 16 & 50 & 1.0\end{array}$

regular reinforcement of the lever press response. The Ss were then divided into three groups with two animals in each group. Each group was assigned a different value of the lower bound of the reinforced IRT interval. The width of the interval was equal to its lower bound. These values and other procedural details are shown in Table I.

\section{Results and Discussion}

The data, shown in Fig. 1, are presented in the form suggested by Hodos (1963). The relationship between these measures and the overall shape of the IRT distribution is also shown by Hodos. For examples

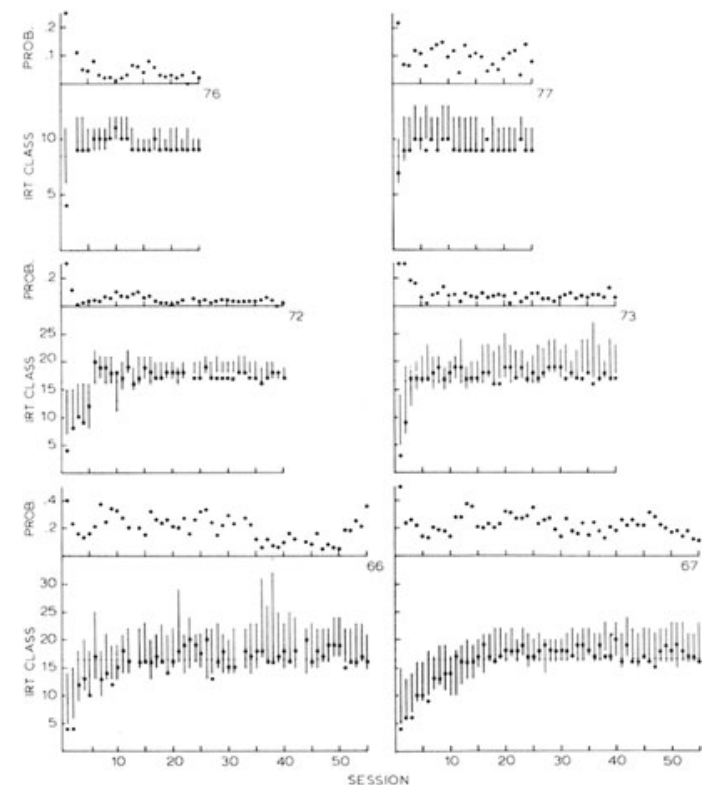

Fig. 1. Measures of the IRT distribution. For details of the procedure relevant to this Fig., consult Table I. The curves for which the ordinate is labeled "Prob." show the probability that the IRT falls within the first three IRT class intervals. The curves for which the ordinate is labeled "IRT Class" show the mode and the semiinterquartile range; the first three IRT classes were omitted in the computation of this later measure. 
of the stabilized form of IRT distributions obtained with these schedules see Malott \& Cumming (1964). It can be seen that the mode of the IRT distribution was initially at a low value and, after several sessions, stabilized slightly above the lower bound of the reinforcement interval. In most cases, the interquartile range was initially below the lower bound; but in every case, it eventually stabilized so that the third quartile was considerably above the lower bound and the first quartile was near the lower bound. The duration of the transition phase for these measures was a negative function of the lower bound. The probability of short IRTs decreased from a high to an intermediate value and stablized more rapidly than the other measures. This result serves to illustrate the point that the transition phase may be different for different response measures; although one measure may have stabilized, another may still be in transition. It is difficult to specify the exact point at which stability was obtained; however, an examination of Fig. 1 indicates that it has been attained by session four for the $4 \mathrm{sec}$. lower bound group, session eight for the $8 \mathrm{sec}$.group, and session 16 for the $16 \mathrm{sec}$. group. It is hoped that these values may serve, at least, as a rough guide in conducting future experiments in this area. These values should be of more general interest since the presence of the upper bound on the interval of reinforced IRTs probably had no effect on the IRT distributions; for Malott \& Cumming (1964) have shown that the steady state distributions obtained with reinforcement interval widths equal to the lower bound are the same as distributions obtained with no upper bound.

\section{References}

CUMMING, W. W., \& SCHOENFELD, W. N. Behavior stability under extended exposure to a time-correlated reinforcement contingency. J. exp. Anal. Behav., 1960, 3, 71-82.

HODOS, W. A simple method for the description of interresponse time distributions. J. exp. Anal. Behav., 1963, 6, 90 .

MALOTT, R. W., \& CUMMING, W. W. Schedules of interresponse time reinforcement. Psychol. Rec., 1964, 14, 211-252.

SIDMAN, M. Tactics of scientific research. New York: Basic Books, 1960.

Notes

1. This research was supported by Public Health Service Grant MH-06911-01.

2. Now at Denison University, Granville, Ohio.

\section{Reply}

The "unspecified 1965 pretraining conditions" to which Battig refers merely involved a gradual increase in the ratio requirement from FR 1 to FR 50 over a 2-day period. As for the 1964 pretraining procedure, it is unlikely that secondary reinforcing properties could have been attached to a stimulus which was associated exclusively with extinction, i.e., the TO stimulus.
The reader is invited to refer to the specific details of this discrimination procedure ( $\mathrm{J}$. exp. Anal. Behav., $1964,7,1-8)$. In any case, the fact that the 1964 results were replicated when the discrimination training procedure was not used seems to rule out a secondary reinforcement interpretation.

Donald M. Thompson University of Virginia

(See page 250 for comment by W. F. Battig) 